\title{
Analysis of Blood Pressure Pulse Wave and Electrocardiogram Waveforms Measured by a Wearable Device with MEMS Sensors
}

\author{
Yosuke Osawa, ${ }^{*}$ Satoshi Hata, Masataka Hori, and Tetsuji Dohi \\ Micro System Laboratory, Chuo University, 1-13-27, Kasuga, Bunkyo-ku, Tokyo 112-8551, Japan
}

(Received October 8, 2020; accepted December 10, 2020)

Keywords: MEMS three-axis force sensor, tonometry, wearable device, blood pressure pulse wave, electrocardiogram

The aim of this study was to clarify which elements of a blood pressure pulse wave are related to cardiovascular diseases and which are not. We developed a device consisting of micro-electromechanical-systems (MEMS) three-axis force sensors, an electrocardiograph, a signal processing board, and a fixed band. It measures blood pressure using force sensors that are placed on the surface of the skin over a blood vessel. During experimentation, blood pressure was varied by breath holding while simultaneously measuring the blood pressure pulse wave and the electrocardiogram (ECG). Furthermore, features derived from the blood pressure pulse wave, its differential waveforms, such as the acceleration pulse wave, and the ECG were compared. The features could be classified according to blood pressure and correlation. The correlation coefficient between the pulse pressure from the blood pressure pulse wave and the $\mathrm{P}$ wave amplitude of the ECG, associated with hypertension, was 0.976 . Moreover, the correlation coefficient between the augmentation index of the blood pressure pulse wave and the ST segment elevation of the ECG, which is used for the diagnosis of myocardial infarction, was 0.915. Thus, we were able to identify features associated with the blood pressure pulse wave and ECG that could diagnose cardiovascular diseases.

\section{Introduction}

Diseases of the circulatory system, such as cerebral vascular disease and heart disease, are often reported as having a sudden onset as well as causing rapid changes in symptoms already present. ${ }^{(1)}$ Therefore, it is of paramount importance to acquire daily biological data. Moreover, symptoms such as the development of hypertension or a rapid change in blood pressure may increase the risk of fatal diseases such as myocardial infarction and stroke. ${ }^{(2)}$ Frequent and continuous blood pressure measurements are important for the efficient diagnosis and treatment of such cardiovascular diseases. Ambulatory blood pressure monitoring (ABPM), which measures blood pressure hourly using an upper-arm cuff, is known to place a heavy burden on its users and to deliver a discontinuous blood pressure measurement. Moreover,

*Corresponding author: e-mail: osawa@msl.mech.chuo-u.ac.jp

https://doi.org/10.18494/SAM.2021.3142 
the Holter electrocardiogram (ECG), which takes an ECG measurement over a span of $24 \mathrm{~h}$ that can be used in the diagnosis of heart disease, has been deployed in the medical field. On the other hand, skin rashes have been reported as a result of applying electrodes to the skin, and the measurement is limited to only one day. Although with a wearable device, a subject's pulse wave and breath can be simultaneously measured, ${ }^{(3)}$ no attempts to measure vital signs associated with cardiovascular disease have been reported.

We have studied the blood pressure pulse wave using the arterial tonometry method, ${ }^{(4)}$ which measures blood pressure with minimal invasiveness. A typical device using this method has been developed by Omron Co., Ltd. ${ }^{(5)}$ The device is unique in that it has two rows of pressure sensor arrays and can be automatically adjusted to an appropriate wearing angle based on values measured from pressure sensors. However, the device is too large to be worn in daily life and does not eliminate noise originating from body movement. Therefore, Fukahori et al. proposed a calibration algorithm that can measure the blood pressure pulse wave during exercise by placing force sensors that measure noise due to body movement and compensate for the blood pressure. ${ }^{(6)}$ The algorithm reduced the standard deviation of the amplitude by $72.0 \%$ when measuring the blood pressure pulse wave while moving the wrist back and forth. It is also hypothesized that the blood pressure pulse wave shares many features with the ECG, which gauges cardiac malfunctions, because the blood pressure pulse wave is measured from the heartbeat. In a previous study, the blood pressure pulse wave and the ECG were simultaneously measured by arterial tonometry, ${ }^{(7)}$ revealing the similarity between the continuously measured blood pressure pulse wave and the ECG. Thus, an algorithm to convert the blood pressure pulse wave to the ECG was proposed. However, it is thought that the blood pressure pulse wave and the ECG have some information in common and both related and unrelated information. In this study, we aim to clarify which elements of a blood pressure pulse wave are related to cardiovascular diseases and which are not.

\section{Principle and Device}

\subsection{Principle of arterial tonometry}

Arterial tonometry can be used to continuously measure blood pressure. ${ }^{(8)}$ Figure 1 shows the principle of the method for measuring the blood pressure pulse wave, as carried out in this

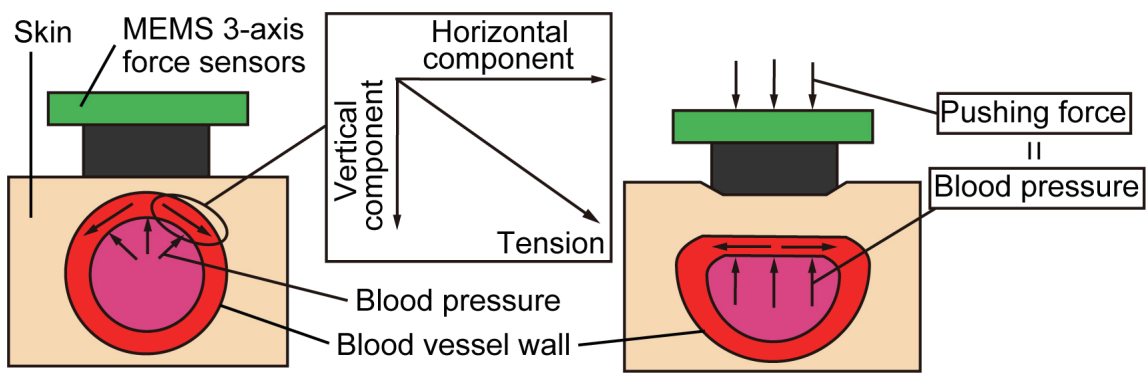

(a)

(b)

Fig. 1. (Color online) Principle of arterial tonometry. (a) Without pushing force. (b) With moderate pushing force. 
study. In this method, blood pressure is measured by a micro-electro-mechanical-systems (MEMS) three-axis force sensor placed on the surface of the skin over an artery. Under the condition shown in Fig. 1(a), the measured pressure differs from the blood pressure because the measured pressure includes the vertical component of the tension on the blood vessel wall. As shown in Fig. 1(b), the tension acting on the blood vessel wall only becomes a horizontal component when the upper surface of the blood vessel is flattened by being pushed with a moderate force from the sensor. Since this pushing force is almost equal to the blood pressure, we can measure blood pressure directly with the force sensor. This method can continuously and noninvasively measure blood pressure, unlike the more common oscillometric method.

\subsection{Fabrication process of MEMS three-axis force sensor ${ }^{(9,10)}$}

The principle of the MEMS three-axis force sensor used in this study is shown in Fig. 2. ${ }^{(9)}$ The sensor consists of three pairs of Si sidewall-doped beams, one of which measures the normal stress and the other two measure the shear stress. The beams that measure the shear stress are arranged vertically, and the piezo-resistance layer is placed inside their sides. Since the MEMS three-axis force sensor is embedded in silicone rubber, the beams deform in the direction of the force when a shearing force is applied as shown in Fig. 2(a). When the beams are deformed, the resistance regions formed inside the two pairs are stretched and compressed in the direction of the deformation. Therefore, the change in resistance caused by the deformation of the beams can be detected as a voltage change by a Wheatstone bridge circuit and amplifier circuit. Through joint research with Shinano Kenshi Co. Ltd. and Touchence Inc., we improved this MEMS three-axis force sensor to realize a sensor for blood pressure pulse wave measurement as shown in Fig. 2(b).

\subsection{Fabrication of device for measuring blood pressure pulse wave and ECG}

We fabricated a wristband-type wearable device to measure both the blood pressure pulse wave by arterial tonometry and the ECG. Figure 3 shows the wristband-type device, comprising a MEMS three-axis force sensor array, an electrocardiograph, a fixed band, and a signal processing board. The device was jointly developed with Shinano Kenshi Co. Ltd.

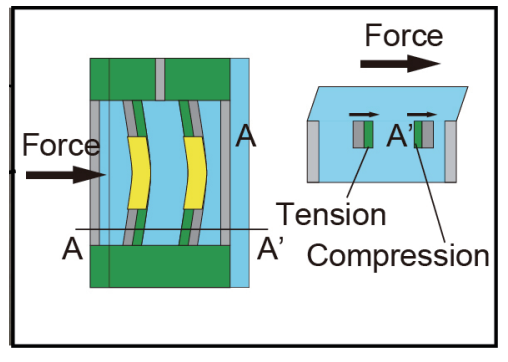

(a)

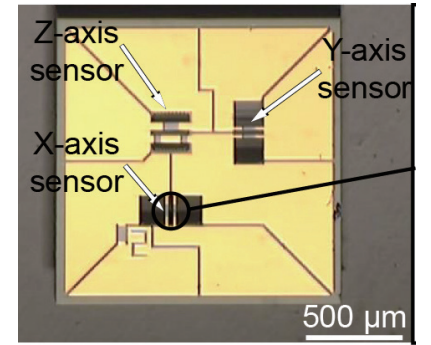

(b)

Fig. 2. (Color online) MEMS three-axis force sensor. ${ }^{(9)}$ (a) Principle of the force sensor. (b) Prototype of the MEMS three-axis force sensor. 


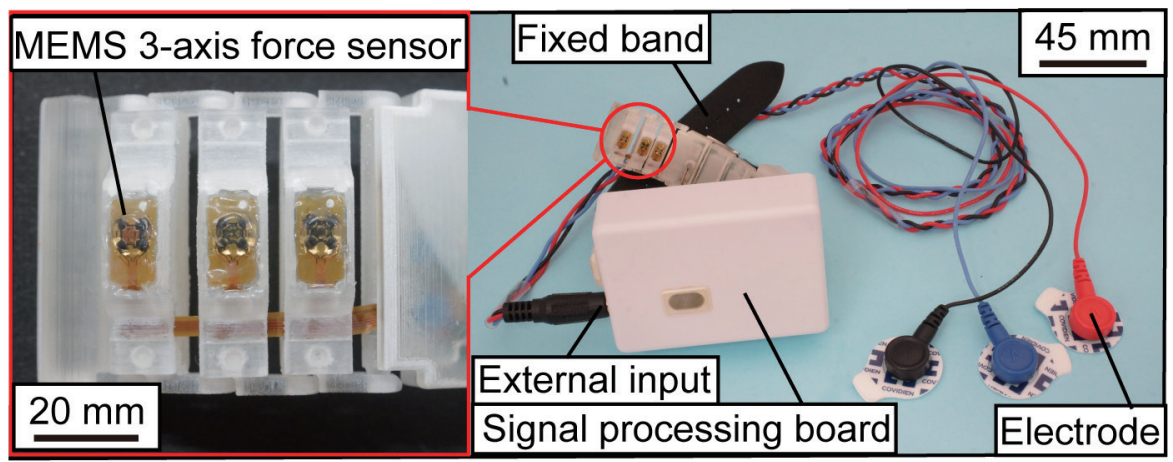

Fig. 3. (Color online) Photograph of wearable device.

The MEMS three-axis force sensor array has three sensing elements and a structure in which a MEMS chip is embedded in silicone rubber. The measured signal is amplified by the signal processing board, and we record the three-axis force on a PC using dedicated software. An ECG sensing board is also attached to the signal processing board, and the ECG can be measured by connecting the cable of an electrocardiograph electrode. The band roughly adjusts the pushing force of the sensor when fixing the device to the wrist. The signal processing board is composed of an amplifier circuit, an analog-to-digital conversion circuit, and a module to transmit measured force data from the sensors.

\section{Experiments and Evaluation}

\subsection{Simultaneous measurement of blood pressure pulse wave and ECG}

While measuring the blood pressure pulse wave and the ECG using the above-described wearable device, we confirmed that feature points could be extracted from the resulting waveforms. The device was fitted by positioning it so that the shear force was zero at the time of fitting and fastening the band so that the amplitude of the blood pressure pulse wave was maximized. Pressure was applied to the sensor by tightening the band of the device, referring to a study in which the suitable pressure was quantitatively measured during wear. ${ }^{(11)}$ As shown in Fig. 4, the device was attached to the left wrist and the positive, negative, and ground electrodes of the ECG were attached to the right clavicle, left clavicle, and right costa, respectively. When we measured the blood pressure pulse wave, the subject's wrist was positioned at the same elevation as his heart. Measurement experiments were started after 1 min rest. A healthy 23 -year-old male subject participated in the measurement.

Figure 5 shows the waveforms of the ECG and blood pressure pulse wave obtained by simultaneous measurement. The standard deviation of the maximum blood pressure pulse wave was $3.38 \mathrm{mmHg}$, which was small enough to confirm that the accuracy of the sensor and the mounting of the device were sufficient to measure the blood pressure pulse wave. As shown in Fig. 6, we extracted the feature points from waveforms of the blood pressure pulse wave, the ECG, the velocity blood pressure, and the acceleration blood pressure. We calculated the 


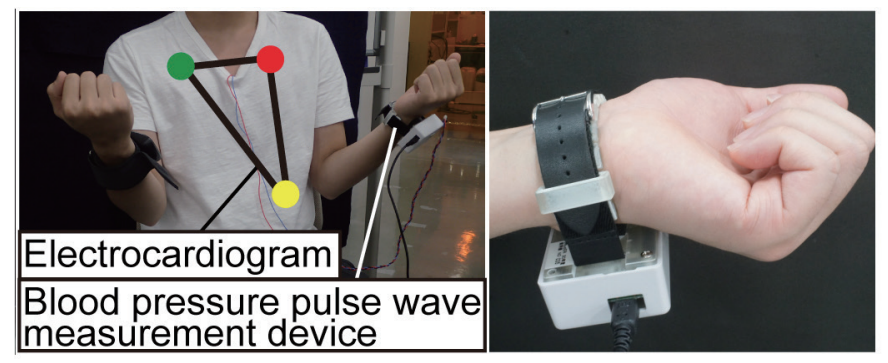

Fig. 4. (Color online) Experimental setup of the simultaneous measurement of the blood pressure pulse wave and the ECG.

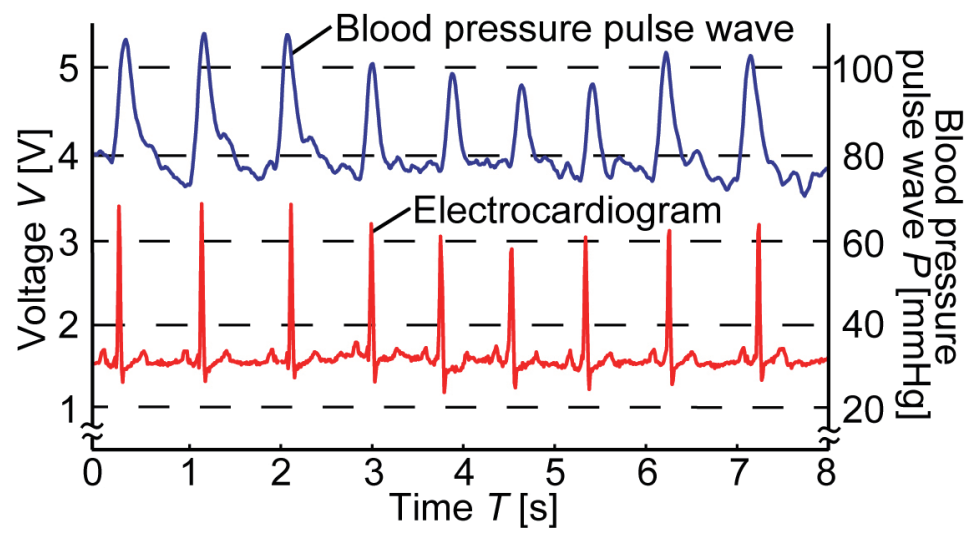

Fig. 5. (Color online) Waveforms of the blood pressure pulse wave and the ECG.

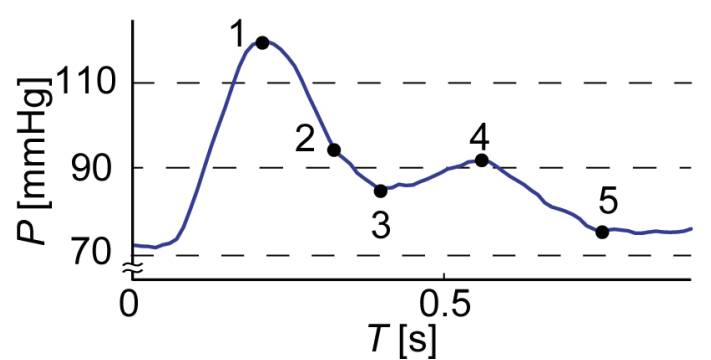

(a)

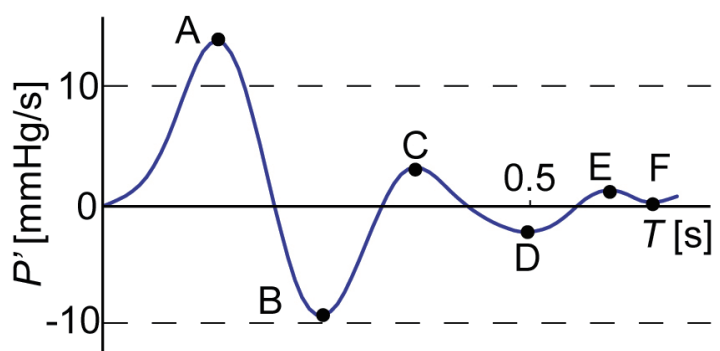

(c)

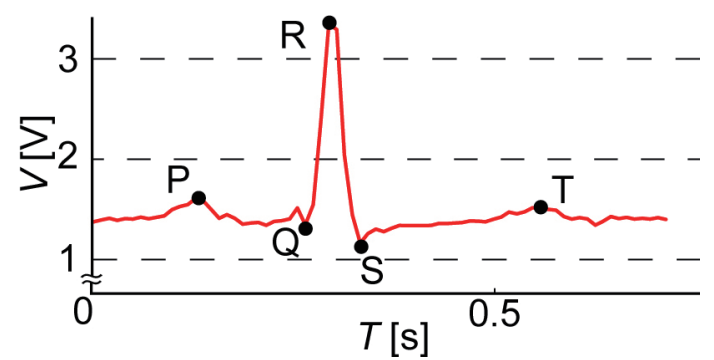

(b)

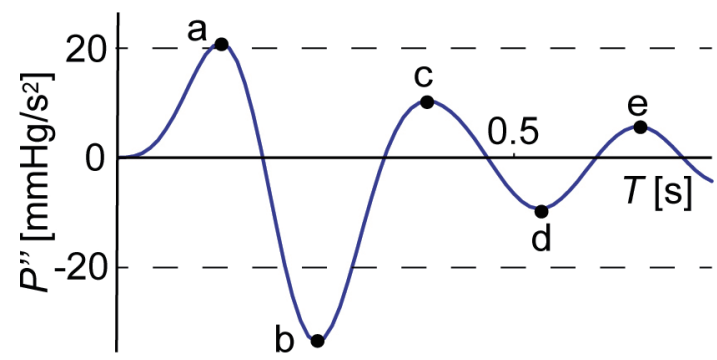

(d)

Fig. 6. (Color online) Feature points of waveforms measured by the wearable device. (a) Blood pressure pulse wave. (b) Electrocardiogram. (c) Velocity blood pressure. (d) Acceleration blood pressure. 
velocity pulse wave in order to observe the inflection point of the blood pressure pulse wave and calculate the acceleration pulse wave. In addition, to clarify the relationship between the ECG and the acceleration pulse wave, which has been reported to be related to vascular stiffness, ${ }^{(12)}$ was also calculated. It was confirmed that feature points could be accurately extracted from the extreme and inflection points of the measured waveforms.

\subsection{Extracted features of the measured waveforms}

The values of the extracted features obtained from the vertical axis are denoted by $A$ followed by a subscript, the time interval given by the horizontal axis is denoted as $I$, and the extracted features are defined using extracted feature points. In addition, the period of the blood pressure pulse wave $\left(H R=I_{5}-I_{5}\right.$, , augmentation index [AIx $=\left(A_{2}-A_{5}\right) /\left(A_{1}-A_{5}\right)$ ], pulse pressure $\left(P P=A_{1}-A_{5}\right)$, mean blood pressure $\left(M B P=A_{5}+P P / 3\right)$, and the ratio of $P P$ to $M B P(R a=P P / M B P)$ were calculated. The features extracted from the acceleration pulse wave were $A_{b} / A_{a}$ and the acceleration pulse graph (APG) index $\left(=\left(A_{c}+A_{d}-A_{b}\right) / A_{a}\right)$. The features obtained from the ECG were the QRS complex $\left(I_{Q R S}\right)$, QT interval $\left(I_{Q T}\right)$, PQ interval $\left(I_{P Q}\right)$, ST segment $\left(I_{S T}\right)$, QRS amplitude $\left(A_{Q R S}\right)$, T wave amplitude $\left(A_{T}\right), \mathrm{P}$ wave amplitude $\left(A_{P}\right)$, Q wave amplitude $\left(A_{Q}\right)$, and ST segment elevation $\left(A_{S T}\right)$. These are medically relevant features that also serve as indicators for pathological analysis. Finally, the pulse transit time (PTT), which is the time difference between the maximum point of the blood pulse wave and that of the ECG, was extracted from the raw data without using a moving average.

\subsection{Blood pressure fluctuation experiment}

A blood pressure fluctuation experiment was performed to compare changes in the extracted features. The device was attached as in the simultaneous measurement of the blood pressure pulse wave and the ECG. In addition, a cuff-type commercial sphygmomanometer was attached to the right wrist for comparison. First, the simultaneous measurement of the blood pressure pulse wave and the ECG began as the subject took a deep breath while seated. Next, the breath holding test, in which blood pressure fluctuated while the breath was held, was performed, and blood pressure was measured using the cuff-type commercial sphygmomanometer. Blood pressure was then measured after having the subject recover by taking a deep breath.

Figure 7 shows the measured blood pressure pulse wave. The parts surrounded by the black circles indicate when blood pressure was measured with the cuff-type commercial sphygmomanometer. Blood pressure was confirmed to have been falling in black circle 1 . The blood pressure then increased after the breath holding test in black circle 2 and decreased when taking a deep breath again (black circle 3). As shown by black circle 4, it was found that the blood pressure at rest was higher than that during deep breathing.

Figure 8 shows the correlation between the measured blood pressure pulse wave and blood pressure. The vertical axis represents blood pressure $P[\mathrm{mmHg}]$ measured by the sphygmomanometer, and the horizontal axis represents force $F[\mathrm{~N}]$ measured by the MEMS three-axis force sensor. Systolic blood pressure $(S B P)$ was measured using the 


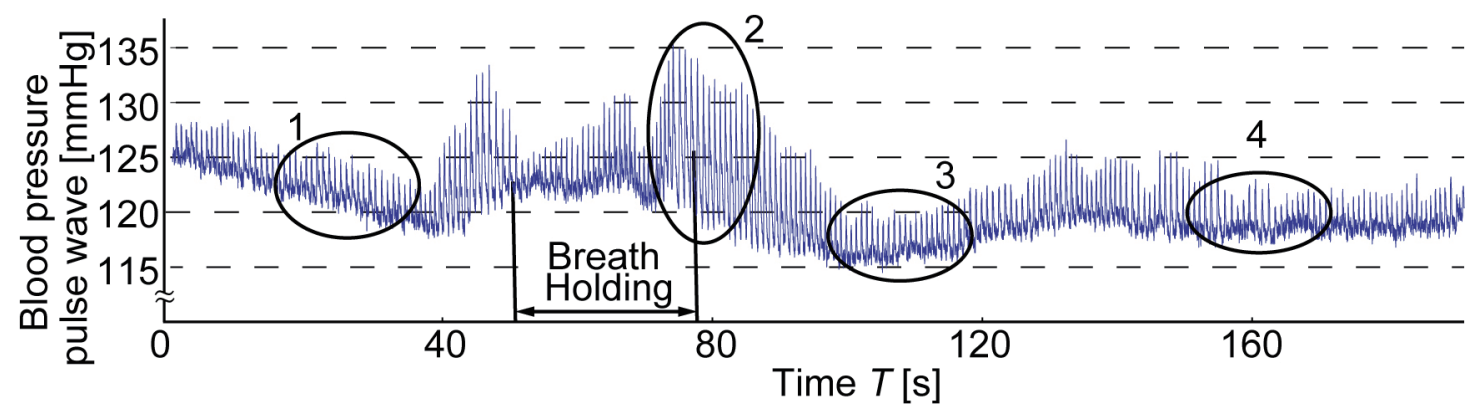

Fig. 7. (Color online) Blood pressure pulse wave during breath holding.

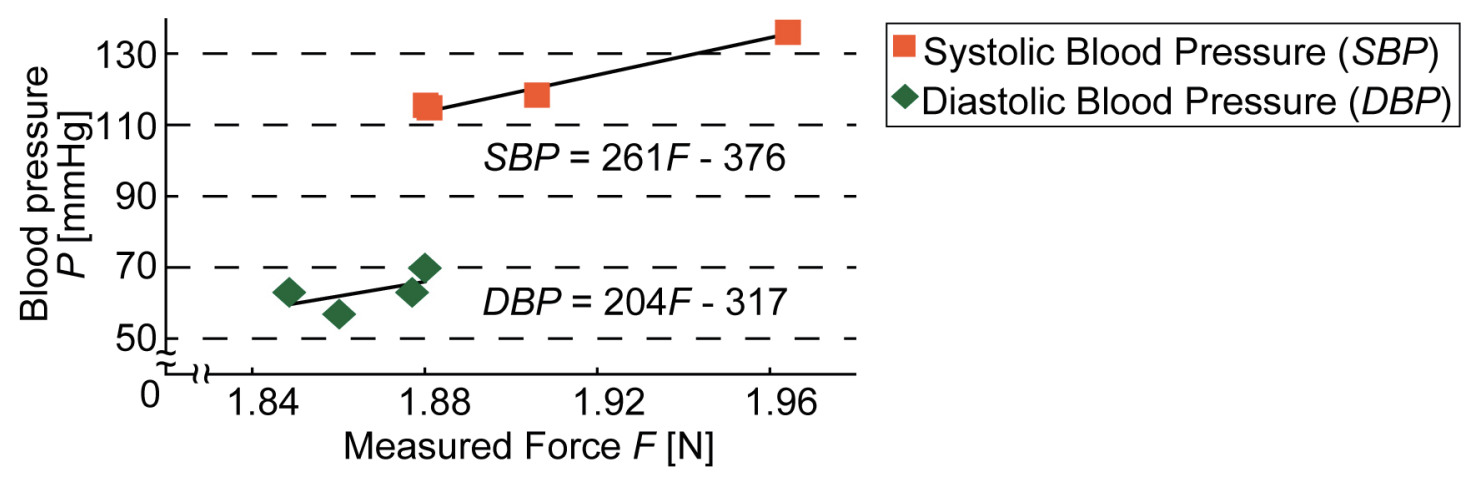

Fig. 8. (Color online) Relationship between the blood pressure and the force measured by a blood pressure pulse wave measurement device.

sphygmomanometer and showed a strong correlation with the maximum value of the blood pressure pulse wave $\left(A_{1}\right)$. A positive correlation was also confirmed between diastolic blood pressure and the minimum value of the blood pressure pulse wave $\left(A_{5}\right)$. Since there was a stronger correlation between $S B P$ and $A_{1}$, the regression formula relating pressure $(P)$ and force $(F)$ shown in Eq. (1) was used as the calibration formula for the blood pressure pulse wave.

$$
P=261 F-376
$$

The force $F[\mathrm{~N}]$ measured by the MEMS three-axis force sensor can be calibrated with the blood pressure pulse wave $P[\mathrm{mmHg}]$ using Eq. (1). The blood pressure pulse wave shown in Figs. 5-7 was calibrated using this regression equation. Table 1 shows the correlation coefficients between $S B P$ and the features of the measured blood pressure pulse wave and ECG. The correlation coefficient between $S B P$ and $A_{1}$ was $R=0.995$. Furthermore, a negative correlation was confirmed between $S B P$ and $\ln (\mathrm{PTT})$. Therefore, it is confirmed that the fluctuation in blood pressure was measured by the device. 
Table 1

Correlation between systolic blood pressure and features of measured waveforms.

\begin{tabular}{lcllc}
\hline Feature & Correlation coefficient & & Feature & Correlation coefficient \\
\cline { 1 - 2 } \cline { 1 - 2 }$A_{1}$ & 0.995 & & PTT & -0.510 \\
$A_{34}$ & 0.068 & & $\ln (\mathrm{PTT})$ & -0.511 \\
$P P$ & 0.942 & & $A_{P}$ & 0.992 \\
AIx & -0.609 & & $A_{Q R S}$ & -0.403 \\
$R a$ & -0.737 & & $A_{S T}$ & -0.462 \\
$I_{13}$ & 0.917 & & $A_{T}$ & -0.957 \\
$I_{23}$ & 0.380 & & $A_{Q}$ & 0.577 \\
$H R$ & -0.971 & & $I_{P Q}$ & -0.856 \\
$A_{B F}$ & -0.254 & & $I_{Q R S}$ & 0.933 \\
$A_{b} / A_{a}$ & -0.933 & & $I_{Q T}$ & 0.716 \\
APGindex & 0.820 & $I_{S T}$ & -0.0825 \\
\hline
\end{tabular}

\section{Discussion}

As shown in Fig. 7, blood pressure gradually decreased in black circle 1. This is thought to be because the parasympathetic nerve system became dominant due to deep breathing. In black circle 2, fluctuation of the commonly known waveforms was noted when the breath holding test was performed. ${ }^{(13)}$ The blood pressure gradually dropped after deep breathing in black circle 3 , and black circle 4 showed a rise in blood pressure because the sympathetic nerve system then became dominant at rest.

Regarding the extracted features, AIx, which is the ratio of the reflected wave to the ejection wave, is related to arterial stiffness. ${ }^{(14)}$ Moreover, MBP represents total peripheral resistance. The ratio of $P P$ to $M B P$ is the ratio of arterial stiffness to total peripheral resistance. Since these features can be used to evaluate the characteristics of blood vessels, it is presumed that they are related to diseases of the circulatory system. ${ }^{(15)}$

The experimental results showed that the blood pressure pulse wave and the ECG features were classified into those that changed with blood pressure and those that did not. Table 2 shows the results of a comparison between the features having a correlation coefficient higher than 0.7 with $S B P$ in Table 1. Table 3 shows the results of a comparison between the features having a correlation coefficient lower than 0.7 with $S B P$ in Table 1.

Features that change with blood pressure are considered to be associated with blood pressure. In fact, $P P$ and $A_{P}$, which are associated with hypertension, appear in Table 2 and show a strong correlation with each other. Moreover, $A_{T}$ might be negative in the case of hypertension or cardiomyopathy, and it showed a strong correlation with $A_{b} / A_{a}$, APG index, and $R a$, indicating the elasticity of the blood vessels. Thus, we were able to extract features associated with blood pressure from the blood pressure pulse wave and the ECG.

Finally, features having a weak correlation with $S B P$ were compared. Although these features are not related to blood pressure, those that showed a strong correlation are shown in Table 3. This result suggests that the blood pressure pulse wave is a factor of waveform variations that are not related to blood pressure. A strong correlation was confirmed between $A_{S T}$, which might be associated with myocardial infarction, and AIx. Elevated $A_{S T}$ is known 
Table 2

Correlation between features strongly correlated with systolic blood pressure.

\begin{tabular}{lcccc}
\hline Feature & Correlation coefficient & & Feature & Correlation coefficient \\
\cline { 1 - 2 } \cline { 5 - 6 }$A_{P}-P P$ & 0.976 & & $A_{T}-A_{b} / A_{a}$ & 0.943 \\
$A_{T}-R a$ & 0.769 & & $A_{T}-$ APGindex & -0.882 \\
$I_{Q T}-H R$ & -0.574 & & $I_{P Q}-I_{13}$ & 0.924 \\
\hline
\end{tabular}

Table 3

Correlation between features weakly correlated with systolic blood pressure.

\begin{tabular}{lcccc}
\hline Feature & Correlation coefficient & & Feature & Correlation coefficient \\
\cline { 1 - 2 } \cline { 5 - 5 }$A_{S T}-\mathrm{AIx}$ & 0.915 & & $A_{Q R S}-A_{B F}$ & 0.980 \\
$A_{Q}-A_{34}$ & 0.748 & & $I_{S T}-I_{23}$ & -0.863 \\
\hline
\end{tabular}

to be attributable to arteriosclerosis as a major factor in myocardial infarction, and the result showed a strong relationship with AIx, indicating arterial stiffness. From the above results, we confirmed the features that show a strong relationship between the blood pressure pulse wave and the ECG. Thus, it is suggested that such features of the blood pressure pulse wave can be used as indexes to evaluate cardiovascular health.

\section{Conclusion}

In this study, the blood pressure pulse wave and the ECG were simultaneously measured by arterial tonometry. Since the standard deviation of the maximum value of the blood pressure pulse wave was $3.38 \mathrm{mmHg}$, blood pressure could also be measured with high accuracy. Moreover, features associated with vital signs were extracted from the measured waveforms. After the changes in blood pressure were confirmed using a fabricated wearable device in a blood pressure fluctuation experiment, correlations among the obtained features were compared. The correlation coefficient between the pulse pressure from the blood pressure pulse wave and the $\mathrm{P}$ wave amplitude of the ECG, which is used for the diagnosis of hypertension, was 0.976 . In addition, the correlation coefficient between the augmentation index of the blood pressure pulse wave and the ST segment elevation of the ECG, which is used for the diagnosis of myocardial infarction, was 0.915 . On the basis of these results, we confirmed the features of the blood pressure pulse wave associated with the ECG that could be used to diagnose cardiovascular disease. Therefore, it is suggested that the features can be used as indexes to evaluate cardiovascular health.

\section{References}

1 J. Dunn, R. Runge, and M. Snyder: Personalized Med. 15 (2018) 1. https://doi.org/10.2217/PME.2018.0044

2 B. M. Psaty, M. Cushman, and R. N. Bryan: Am. Med. Assoc. 161 (2001) 1183. https://doi.org/10.1001/ ARCHINTE.161.9.1183

3 T. Nguyen and M. Ichiki: Sensors 19 (2019) 4942. https://doi.org/10.3390/s1922494

4 T. Dohi and K. Waki: Proc. Int. Symp. Micro-Nano Sci. Technol. 2016 (2016) SuA1-B-8. 
5 Y. Kato and T. Hamaguchi: Proc. OMRON TECHNICS (2018) 33 (in Japanese).

6 S. Fukahori, M. Hori, and T. Dohi: Proc. Symp. Micro-Nano Sci. Technol. 2017 (2017) 02pm1-PN-127 (in Japanese).

7 S. Sato, M. Hori, and T. Dohi: Proc. Biomedical Engineering Society Kanto Branch Young Researcher Conf. (2018) C-2-04 (in Japanese).

8 O. Kemmotsu, M. Ueda, H. Otsuka, T. Yamamura, A. Okamura, T. Ishikawa, D. C. Winter, and J. S. Eckerle: Anesthesia Analgesia 73 (1991) 54. https://doi.org/10.1213/00000539-199107000-00011

9 H. Takahashi, A. Nakai, N. Thanh-Vinh, K. Matsumoto, and I. Simoyama: Sens. Actuators, A 199 (2013) 43. https://doi.org/10.1016/j.sna.2013.05.002

10 R. Hino, M. Hori, and T. Dohi: Proc. Bioengineering Conf. Papers (2015) 2 C32 (in Japanese).

11 M. Sato, M. Hori, and T. Dohi: Proc. Robotics and Mechatronics Conf. 2016 (2016) 2P1-02b7 (in Japanese).

12 K. Fujimoto, Y. Sano, and E. Watanabe: Human Eng. 48 (2012) 285. https://doi.org/10.5100/jje.48.285 (in Japanese)

13 L. Pstras, K. Thomaseth, J. Waniewski, I. Balzani, and F. Bellavera: Acta Physiol. 217 (2016) 1. https://doi. org/10.1111/apha.12639

14 I. B. Wilkinson, K. Prasad, I. R. Hall, A. Thomas, H. MacCallum, D. J. Webb, M. P. Frenneaux, and J. R. Cockcroft: J. Am. College Cardiol. 39 (2002) 1005. https://doi.org/10.1016/S0735-1097(02)01723-0

15 T. Ozawa: Arterial Stiffness 8 (2012) 285 (in Japanese). http://www.arterial-stiffness.com/pdf/no08/009 015. pdf 